\title{
Main Bacterial Peritonitis Infection Caused by Streptococcus pneumoniae
}

\author{
Sofia Maraki, "1, Panagiotis Moraitis, Sophia Barbagadakis, Ioannis Vlachakis \\ ${ }^{1}$ Departments of Clinical Microbiology and Microbial Pathogenesis and Pediatric Surgery, University Hospital of \\ Heraklion, Crete, Greece
}

Corresponding author: Dr. Sofia Maraki, Department of Clinical Microbiology and Microbial Pathogenesis, University Hospital of Heraklion, Heraklion 71110, Crete, Greece. E-mail: sofiamaraki@ yahoo.gr

Citation: Maraki S, Moraitis P, Barbagadakis S, Vlachakis I. Main bacterial peritonitis infection caused by Streptococcus pneumonia published in Med Science Journals.

Received Date: 10 July 2021 Accepted Date: 27 July 2021 Published Date: 13 August 2021

\begin{abstract}
Primary peritonitis is a rare infection in healthy children, mainly affecting patients with underlying medical disorders. We report a case of primary pneumococcal peritonitis in an immunocompetent 3-year-old boy. The analysis and diagnosis was made at laparotomy and cultures of the intra-abdominal pus yielded Streptococcus pneumoniae. Timely antibiotic treatment administered resulted in complete resolution of the infection.
\end{abstract}

Keywords: Antimicrobial treatment, children, diagnosis, primary peritonitis, Streptococcus pneumoniae

\section{Introduction}

Primary or spontaneous peritonitis is outlined as Associate in Nursing infection of the greater peritoneal sac while not a plain a plain. within the within the primary redness accounted for 100 percent of all abdominal emergencies in youngsters, then again with the arrival of antibiotics, its incidence attenuated to $1 \%-2 \%$. Spontaneous diplococcus redness in youngsters has been recognized for over a century. It happens at any age, however its peak incidence is between four and nine years ancient, and it's a lot of common in women than boys (ratio, 4:1). within the gift article, we tend to describe a case of primary diplococcus redness in a very antecedently healthy boy, and that we review the literature on this infection.

\section{Case Report}

A 3-year-old boy was delivered to the emergency department attributable to attributable to history of abdominal pain, nausea, anorexia, and fever. His anamnesis was routine. On admission, his pulse was a hundred thirty-five M.M., his temperature was $38^{\circ} \mathrm{C}$, and his pressure was $101 / 81 \mathrm{mmHg}$. The physical examination of the abdomen discovered diffuse tenderness, soreness, guarding, and diminished intestine sounds. Abdominal ultrasound detected exaggerated amounts of serous membrane fluid. Chest hearing didn't reveal any pathologic signs and chest radiography was traditional. In addition, no focal sites of infection on the ear, nose, and throat were detected.

Laboratory results showed associate elevated peripheral white somatic cell (WBC) count of twelve,200/mm3 (neutrophils $75 \%$ ), and a C-reactive supermolecule elevated at four. $5 \mathrm{mg} / \mathrm{dL}$ (normal values, $0.08-0.8 \mathrm{mg} / \mathrm{dL}$ ). different laboratory analyses comprised negative HIV and HCV testing, liver operate tests, urinary organ operate tests, and medicine investigations were at intervals the conventional limits. \{urinalysis|uranalysis/chemical associatealysis|qualitative analysis|diagnosis|diagnosing \} showed an acid $\mathrm{pH}$ of five.5 and relative density of 1026 , while not leukocytes, erythrocytes, crystals, or casts. Blood and excretory product cultures taken on admission yielded no microorganism growth. because of the probationary identification of acute rubor, antimicrobial treatment was by trial and error initiated with endovenous Claforan three hundred mg each vi h, amikacin one hundred mg each eight $h$, and antiprotozoal two hundred mg each eight $\mathrm{h}$, and at intervals a couple of hours from admission, associate preliminary incision was performed. Intraoperatively, pussy secretions were found within the abdomen, however no obvious intraperitoneal lesion might be known. The appendix, that appeared macroscopically traditional, was removed and sent for microscopic anatomy examination. Histology incontestable gentle inflammatory alterations. 
Gram-stained smears of the pussy exudate incontestable incontestable diplococci, and culture yielded eubacteria pneumoniae (serotype 11A), that was intermediately prone to antibiotic drug, however prone to prone to (cefotaxime and ceftriaxone), macrolides, quinolones, tetracyclines, tigecycline, linezolid, and glycopeptides. the kid was absolutely insusceptible against S. pneumoniae with the 13-valent diplococcus conjugate immunogen (PCV13). supported the results of the antimicrobial susceptibleness testing of the isolate, antiprotozoal and amikacin were discontinued. Claforan treatment was continued for a complete of ten days. Over the subsequent days, the patient's condition was improved. The peripheral blood corpuscle counts bit by bit minimized to 7700/mm3 (neutrophils forty.9\%), and C-reactive supermolecule at zero. $35 \mathrm{mg} / \mathrm{dL}$. The patient was discharged cured on the eleventh surgical day.

\section{Discussion}

This is a diagnosed case of primary diplococcus inflammation during a during a with typical symptoms of acute abdomen inflammation. The route of entry of pneumoccci is sometimes not apparent, and sometimes it's plausible to be hematogenous unfold ofttimes from the tract, lymphogenous through transdiaphragmatic lymphatics, or translocation through associate degree intact enteral wall from the gut lumen. In females, the microorganisms might ascend from the canal via the fallopian tubes.

Primary inflammation has been reported not solely in youngsters with postnecrotic cirrhosis of the liver, nephrosis and general LE however conjointly affects otherwise healthy youngsters while not underlying predisposing conditions, as in our case. many decades agone S. pneumoniae and blood group streptococci were the organisms most ordinarily reported to cause primary inflammation. Fowler reported fifty cases of diplococcus primary inflammation from 1925 to 1955 and solely half-dozen cases from 1956 to 1970.

In a during a from 2000 to 2004 in Canadian province, Canada, among 1768 cases of invasive diplococcus illness (IPD) in each adults and youngsters, only $23(1.3 \%)$ cases of inflammation because of S. pneumoniae were found. Of these, solely four concerned youngsters, two of that had a history of nephrosis, one suffered from viral hepatitis and bladder rupture, and only one was a antecedently healthy 2-year-old woman. though diplococcus inflammation declined by the top of the twentieth century, associate degree enhanced incidence of the illness was reported in geographical region, before the introduction of the conjugate diplococcus vaccines in childhood. In Chile, among 491 IPD cases diagnosed in youngsters, 116 (23.6\%) were inflammation, with a transparent predominance of feminine patients (91 ladies, 78.4\%). Among them, solely half-dozen ladies had a predisposing medical condition, whereas ten out of twenty-five male patients with inflammation conjointly suffered from nephrosis. In Central American country among 132 youngsters with IPD, $7.4 \%$ had inflammation.

The last decade, 3 kids, 2 boys and one woman, were diagnosed with primary diplococcus peritoneal inflammation in our establishment. All 3 kids had no underlying predisposing conditions. S. pneumoniae isolated from our patient belonged to serotype $11 \mathrm{~A}$, one amongst the foremost prevailing serotypes in our space once the introduction of the 13-valent diplococcus conjugate immunogen (PCV 13).

\section{Conclusion}

Concluding, primary diplococcus peritoneal inflammation ought to be enclosed within the medical diagnosis of acute abdomen in kids. Gram-stain and culture of intra-abdominal material, collected throughout surgery or by abdominal puncture, area unit the keys to identification and treatment.Declaration of patient consent The authors certify that they need obtained all acceptable patient consent forms. within the kind the Guardian has given his consent for his son's and different clinical data to be according within the journal. The guardian understands that no names and initials are printed and due efforts are created to hide their identity, however obscurity can't be warranted. analysis quality and ethics statement: The authors followed applicable EQUATOR Network (http://www.equator-network.org/) tips, notably the CARE guideline, throughout the conduct of this report.

\section{Financial support and support}

Nil.

\section{Conflicts of interest}

There aren't any conflicts of interest 


\section{References}

1. McDougal WS, Izant RJ Jr, Zollinger RM Jr. Primary peritonitis in infancy and childhood. Ann Surg 1975;181:310-3.

2. Annand WF, Bowen WH. Pneumococcal peritonitis. Lancet 1906;1:1591-7.

3. Freij BJ, VottelerTP, McCracken GH Jr. Primary peritonitis in previously healthy children. Am J Dis Child 1984;138:1058-61.

4. Fowler R. Primary peritonitis: Changing aspects. Aust Paediatr J 1971;7:73-83.

5. Waisman DC, Tyrrell GJ, Kellner JD, Garg S, Marrie TJ. Pneumococcal peritonitis: Still with us and likely to increase in importance. Can J Infect Dis Med Microbiol 2010;21:e23-7.

6. Lagos R, Munoz A, Martin SO, Maldonado A, Hormazabal JC, Blackwelder WC, et al. Age- and serotype-specific pediatric invasive pneumococcal disease: Insight from systematic surveillance in Santiago, Chile. J Infect Dis 2008;198:1809-17.

Submit your next manuscript to Med Science Publishers and benefit from:

$\rightarrow$ Easy online submission process

$\rightarrow$ Rapid peer review process

$\rightarrow$ Online article availability soon after acceptance for Publication

$\rightarrow$ Open access: articles available free online

$\rightarrow$ More accessibility of the articles to the readers/researchers within the field

$\rightarrow$ Better discount on subsequent article submission

Submit your manuscript at

https://medsciencejournals.com/submit-manuscript 